\title{
Responsabilidad social corporativa, gobernanza e instituciones. Armando el rompecabezas
}

\author{
Luis Montaño Hirose \\ Universidad Autónoma Metropolitana, Iztapalapa, México \\ 1mh52@prodigy.net.mx
}

La responsabilidad moral es la más personal e inalienable de las posesiones humanas, y el más preciado de los derechos humanos.

Zygmunt Bauman

Resumen: La Responsabilidad Social Corporativa (RSC) es analizada desde un doble ángulo analítico, la teoría de la gobernanza corporativa y la del análisis institucional. Desde esta perspectiva, se destacan algunos actores institucionales importantes en la evolución de la RSC, como son la gran empresa, la iglesia, las escuelas de negocios y los grupos de empresarios. Se distinguen dos periodos: el primero va desde los inicios de la gran empresa, a finales del siglo XIX, hasta la década de los setenta del siglo pasado, en él predominó la búsqueda de la productividad como mecanismo de mediación social; el segundo se caracteriza por una orientación hacia la rentabilidad, trastocando el orden social e institucional anterior a través de la implementación de modos de gestión que confinan la responsabilidad social a programas meramente formales.

Palabras clave: Responsabilidad Social Corporativa, gobernanza corporativa, instituciones, modos de gestión.

Corporate Social Responsibility, Governance and Institutions: Making the Pieces Fit

Abstract: Corporate Social Responsibility (CSR) is analysed from a dual analytical perspective: the theory of corporate governance and institutional analysis. From this point of view, the paper highlights some important institutional players in the 
evolution of CSR, such as big business, the church, business schools and entrepreneur groups. We consider two periods; the first starts with the beginning of big business in the late nineteenth century and lasts until the 1970s and was characterised by the pursuit of productivity as a social mediation mechanism. The second is characterized by an orientation towards profitability, disrupting the social and institutional order through the implementation of management methods that reduce social responsibility programs to formal ones.

Keywords: Corporate Social Responsibility, corporate governance, institutions, management methods. 


\section{Introducción y planteamiento general}

La Responsabilidad Social Corporativa (RSC) ha llegado a constituir en nuestros días uno de los temas centrales del debate social. Algunos analistas la consideran como un objeto de moda administrativa, al igual que la planeación estratégica o la calidad total. Otros estiman que se trata de un potente dispositivo discursivo que oculta aspectos que atentan contra la sociedad y el medioambiente, al mismo tiempo que promueve la adhesión a proyectos empresariales. Por otro lado, hay quienes, a partir de la supremacía de los mercados, ven en la RSC un instrumento potente, capaz de reducir el peso de las crisis económicas recurrentes y que constituye un nuevo mecanismo de regulación social que se erige en modelo de responsabilidad para el resto de las instituciones sociales. Otros estudiosos observan en ella más bien un mecanismo para aminorar los escándalos provocados por grandes empresas reconocidas internacionalmente. Desde un ángulo crítico, aflora la conciencia del largo plazo, de los recursos naturales no renovables, del deterioro del medioambiente y de los problemas vinculados a los nuevos modos de gestión, como el desempleo estructural, el empleo precario, el suicidio de mandos medios y la violencia psicológica, por mencionar solo algunos ejemplos.

Dicho en otras palabras, la RSC constituye un aspecto fundamental de la vida social, un tema central del debate político y un objeto de estudio extremadamente complejo. Por ello, la RSC ha sido analizada desde muy diversos ángulos, que efectúan distintos recortes de la realidad. De esta manera, encontramos reflexiones que intentan dar cuenta del origen de los programas de RSC, destacan, entre otros, la globalización, la reducción de la presencia del Estado nacional, el desarrollo de nuevas tecnologías de información y comunicación, la importancia creciente del mercado financiero, la caída del bloque socialista, así como, entre otros, la aparición de nuevas formas de regulación «blanda» (Ahme y Brunsson, 2005) que promueven isomorfismos organizacionales (DiMaggio y Powell, 2001). En función de la importancia otorgada a dichas fuentes - o a una combinación de ellas-, se enfatiza la participación de diversos actores sociales, como la gran empresa, las organizaciones internacionales, las instituciones financieras, el Estado, las universidades, las organizaciones no gubernamentales, los trabajadores, los consumidores, etc., destaca en ocasiones el nivel macrosocial y, en otras, el de la interacción personal. Finalmente, las aproximaciones teóricas presentan un comportamiento similar: van desde las visiones más funcionalistas y positivistas, pasando por las de orden crítico, sustentadas en enfoques marxistas, hasta aquellas que se inscriben en diferentes modalidades del constructivismo social. Armar el rompecabezas de la RSC resulta imprescindible, pero no es tarea fácil. En este trabajo se pretende avanzar en dicha labor proponiendo una línea 
de reflexión que intenta entretejer algunos de los aspectos mencionados de la compleja realidad de la responsabilidad social con el desarrollo y transformación de las grandes empresas y de los principales actores institucionales que han participado históricamente en la orientación de su desarrollo, el establecimiento de sus fines y la coordinación de sus complejas actividades.

Asumimos como punto de inicio en este texto que el debate sobre la RSC se inició casi simultáneamente al surgimiento de las grandes empresas en Estados Unidos, bajo la forma de corporación o empresa por acciones, hacia finales de 1800. Con la corporación, una nueva forma institucional comenzó a dibujarse con gran fuerza hasta llegar a constituir en la actualidad una referencia fundamental para el resto de las instituciones (hospital, universidad, gobierno, etc.). La RSC ha conocido a lo largo de los años transformaciones importantes, que, para ser aprehendidas, tienen que ser contextualizadas históricamente; ello no significa, sin embargo, que su amplia difusión se haya realizado asentándose sobre las bases que la particularizan, sino más bien todo lo contrario, como testimonia el hecho de que ha gozado de una gran aceptación internacional, a pesar de que las realidades sociales, económicas y políticas locales presentan diferencias significativas ${ }^{1}$; lo mismo puede decirse acerca de su incorporación en otros ámbitos institucionales ${ }^{2}$. En este contexto, nuestra reflexión se basará en la corriente analítica denominada gobernanza corporativa que se inició con los trabajos de Berle y Means en 1932, y que fue retomada posteriormente por diversos autores con distintas visiones (económica, política y social), como Burnham (1941), Marris (1964) y Galbraith (1967), entre otros. La tesis central común de esta corriente de pensamiento establece que la gran corporación estadounidense se caracteriza por una separación profunda entre la propiedad y el control, producto de la necesidad de contar con profesionales altamente cualificados que puedan atender los problemas complejos de las grandes empresas. Por otro lado, la necesidad de inversiones cuantiosas requirió la acumulación de montos de capital que no podían ser aportados en su totalidad de manera individual, lo que generó la figura legal de la empresa por acciones. Es así como este tipo de empresa, concentradora de grandes montos de capital, dirigida por un nuevo grupo social cada vez más cualificado, dio pie a una nueva forma institucional, que requirió a su vez el

1 Dicho traslado puede ser observado a lo largo de la historia. Sin embargo, la globalización, las nuevas tecnologías y la búsqueda de competitividad y legitimidad han propiciado que sobre todo a partir de la década de los ochenta del siglo pasado, las modas organizacionales hayan adquirido un gran auge (Abrahamson, 1996; Czarniawska, 2005; Montaño, 2000).

2 Es interesante recordar que Taylor señalaba en 1911 que la administración científica poseía un amplio campo de acción, que no se limitaba al estrecho espacio de la fábrica: «Los mismos principios pueden aplicarse con igual fuerza a todas las actividades humanas: a la administración de nuestros hogares; a la de nuestras granjas; a las de los negocios de nuestros artesanos, grandes y pequeños; a la de nuestras iglesias, de nuestras instituciones filantrópicas, de nuestras universidades y de nuestros departamentos de gobierno». (Taylor, 1961: 17). 
surgimiento y desarrollo de una profesión, la administración, como conjunto de saberes especializados, legitimados en los recintos universitarios, y comenzaron a proliferar de esta manera las escuelas de negocios.

A pesar de la importancia concedida al estudio de los planteamientos teóricos de la gobernanza corporativa para lograr una mejor comprensión de la RSC, estimamos que dicho cuerpo teórico es insuficiente, ya que se trata de una visión preponderantemente económica, que no toma en cuenta ni los aspectos sociales ni los fenómenos organizacionales que ocurren en el interior de las corporaciones ${ }^{3}$. Para subsanar, al menos parcialmente, esta situación, proponemos incorporar dos conceptos. El primero es el de institución, desde nuestro punto de vista, este se encuentra en el cruce de lo económico, lo político, lo social y lo psicológico y constituye una referencia fundamental, aunque ambigua y contradictoria, en el establecimiento del sentido social de las organizaciones y, por lo tanto, en cómo es asumida su responsabilidad social. El segundo de ellos es el de modo de gestión, que puede ser entendido, grosso modo, como un instrumento potente para el logro de los fines institucionales. Discutiremos la creciente separación entre ambos conceptos - institución y modos de gestión - en el marco de la evolución reciente de la gobernanza corporativa y su influencia en el resto de las instituciones sociales, que genera graves crisis de legitimidad.

El texto está compuesto por cinco apartados. En el primero se presentan algunos aspectos teóricos del concepto de institución, así como su relación con la noción de modo de gestión, lo que permitirá contar con elementos de análisis para una mejor comprensión de la línea de reflexión propuesta en este documento. A continuación, se aborda el tema del origen y desarrollo de la gran empresa, una institución particular caracterizada por la separación entre propiedad y control. En el tercer apartado se discute la importancia y vigencia de la iglesia, sobre todo la derivada del movimiento protestante, en cuanto que modalidad institucional, en el desarrollo y orientación de la empresa moderna y su relevancia en las particularidades que aporta al tema de la responsabilidad social. Posteriormente, se analizan algunos aspectos relevantes del saber especializado que ha coevolucionado con la institución empresarial; se hace referencia a las escuelas de negocios y, por lo tanto, a la administración en cuanto que disciplina universitaria. A continuación, se aborda la ruptura entre dos modelos de regulación social, transitando

3 A ello habría que agregar que la diferencia entre ambos grupos, los mánager y los accionistas, no resulta tan clara como ha sido planteada por la corriente teórica de la gobernanza corporativa, ya que existen algunos intereses que son comunes, como puede ser la productividad como forma, no exclusiva, para lograr la rentabilidad así como procesos de negociación que permiten encontrar espacios de interés común como pudiera ser en ocasiones la búsqueda de la rentabilidad a medio plazo. Algunos autores han llegado incluso a cuestionar abiertamente que dicha separación en realidad se haya realizado o que, en todo caso, se requiere mayor evidencia empírica que la constate. En este contexto de debate, también se ha cuestionado el verdadero papel social de su función (Zeitlin: 1974). 
de uno centrado en la productividad a otro basado en la rentabilidad, se analizan las dificultades que entraña en este proceso el discurso de la responsabilidad corporativa.

\section{Institución y modos de gestión. Un acercamiento teórico}

El concepto de institución es muy utilizado en la actualidad y ha sido retomado en diversas discusiones tanto políticas como académicas. Se encuentra en el corazón mismo de planteamientos teóricos tan diversos como el nuevo institucionalismo sociológico (DiMaggio y Powell, 2001) y el económico (North, 1993; Williamson, 1989), y constituye en ambos casos un recurso central para el establecimiento de diversos organismos reguladores y políticas públicas. Tan vasta plasticidad semántica proviene seguramente de su alta complejidad como fenómeno social y de que su «verdadero» significado constituye, por lo tanto, un objeto de disputa por parte de diversos actores sociales. De cualquier manera, sin entrar en profundos detalles, quisiéramos señalar que la aproximación conceptual a la que nos adherimos se inspira en la línea de pensamiento francesa desarrollada por Loureau (1975), Castoriadis (1982; 2006) y Enriquez (1992), aunque obligadamente recurre también a los desarrollos del nuevo institucionalismo sociológico, sobre todo en su vertiente más fenomenológica, la denominada construcción social de la realidad; nos referimos centralmente a los trabajos de Friedland y Alford (2001), así como a los de Berger y Luckmann (1972).

El término institución, en su forma más extendida, y ampliamente utilizada por el nuevo institucionalismo económico, posee un significado muy general: el de las reglas del juego. Estas son impuestas por organizaciones que tienen un peso considerable en la vida social, económica y política al regular el comportamiento de actores que presumen que sus decisiones se rigen por una racionalidad instrumental limitada y egoísta. Una segunda versión sostiene que las instituciones son organizaciones relevantes para el individuo porque participan en aspectos fundamentales de su constitución como entes sociales, destacan, entre otros, los casos de la familia, la escuela y el trabajo. Si nos remontamos a una de sus primeras definiciones, encontramos que fue Emile Durkheim (1894) el primero en referirse a dicho concepto, aunque de manera muy general, al proponer que la institución no es sino un «hecho social» y, por lo tanto, la sociología es en realidad la ciencia de las instituciones. Loureau fue uno de los primeros en tratar a fondo el tema de la institución, arguyendo que se trata de un concepto en constante tensión debido al juego de lo ya «instituido» frente a las fuerzas del cambio, es decir, 
lo «instituyente». Castoriadis, por su parte, considera que la institución es en realidad una red simbólica en la que participan un componente funcional y otro imaginario. Considera que la institución es uno de los dos ingredientes básicos de la humanización, el otro es la imaginación. Lo primero que se instituye, postula el autor, es la sociedad, que forma a los individuos — «fragmentos ambulantes de la institución»-y les proporciona sentido social. A este primer momento lo llama institución primaria: «la sociedad se crea a sí misma» y, para ello, necesita instituciones segundas. Estas pueden ser «transhistóricas», como el lenguaje, el individuo y la familia, o bien «específicas», como la empresa capitalista, y menciona que «la empresa capitalista es [...] una institución segunda específica [...]. No hay capitalismo sin empresa y no existe verdaderamente lo que entendemos por empresa en las sociedades anteriores al capitalismo: esta institución que conlleva una significación, este conjunto de dispositivos y de reglas que reúne a gran cantidad de gente y les impone la utilización de ciertas herramientas y máquinas, controla y organiza jerárquicamente su trabajo, cuya finalidad es el autocrecimiento indefinido» (Castoriadis, 2006: 124).

Por otro lado, la institución es, de acuerdo con Berger y Luckman (1972), la parte final de un proceso de interiorización, que es construido socialmente mediante el uso de convencionalismos, y constituye un poderoso sistema de control; de esta manera, el papel social del individuo se encuentra cifrado en el de institución. Para Enriquez, la institución constituye una de las instancias del funcionamiento de las organizaciones y se refiere a ella como un dispositivo de regulación del orden social que antecede la formación misma de las organizaciones. La institución, de acuerdo con este autor, requiere de un saber especializado para legitimarse socialmente; este se conforma de normas y principios que deben ser difundidos e interiorizados. Finalmente, en esta apretada síntesis, para Friedland y Alford, desde el nuevo institucionalismo sociológico, la institución constituye una referencia supraorganizacional fundamental al dotar a la organización de sentido social.

Tomando en consideración lo expuesto anteriormente, se puede decir que la institución puede ser entendida como una representación mental, idealizada y parcial del orden social, que sintetiza tanto anhelos como temores de una colectividad y proporciona certeza social a la existencia de las organizaciones y sentido a la acción de los individuos (Montaño, 2012a). Las instituciones constituyen el terreno propicio para el desarrollo y funcionamiento de las organizaciones; estas son, de acuerdo con Enriquez, «instituciones hechas fierro». A pesar de las particularidades de cada institución — familia, empresa, escuela, etc.—, estas no funcionan de manera autónoma ni independiente, sino que se compenetran 
mutuamente, coevolucionando, complementándose y contradiciéndose al mismo tiempo; este mismo juego de tensiones lo encontramos de hecho en el interior de cada una de ellas (Friedland y Alford, 1999; Rendón, 2007). Además, las instituciones son, como ya hemos mencionado, la síntesis de las fuerzas instituidas e instituyentes, lo que las hace dinámicas y objeto de querella. La trama social se conforma de este entreveramiento institucional que constituye el escenario en el que actúan las organizaciones y en el que ubican su sentido de responsabilidad social.

Si bien es cierto que las sociedades modernas tienen la capacidad de controlar parte de su desarrollo, esto no significa que los principios, valores y comportamientos asentados en instituciones de tipo tradicional se pierdan totalmente en el pasado (Montaño, 2004). La idea de que la modernidad es la negación, o el sustituto, de la tradición es una idea moderna. Weber (1991) postuló que la tradición calvinista propició el desarrollo de la modernidad empresarial, pero que ese período de cohabitación duró en realidad pocos años, y venció la modernidad. Durkheim (2000) propone, por su lado, que la tradición se entremezcla con la modernidad, dando paso así a la idea de que la ciencia y la religión, a pesar de sus oposiciones, logran establecer formas de convivencia adecuadas.

Por otro lado, el ámbito institucional no es producto exclusivo de la racionalidad y las instituciones evolucionan, transformándose en la escena social. Su entramado presenta un alto nivel de complejidad y tensión, ya que en cada una de ellas podemos encontrar, de manera implícita o explícita, distintas interpretaciones de su sentido social. La institución de la sociedad implica la diversidad de instituciones que la conforman, a pesar de sus paradojas. Cada forma institucional - escuela, empresa, hospital, prisión, etc. - constituye en sí un discurso que requiere un saber y dispositivos particulares para realizar sus pretensiones. Cada una de ellas lleva implícita una noción propia de responsabilidad social. A título de ejemplo, se puede decir que el hospital está pensado para salvar vidas y acabar con la enfermedad y el dolor, o que la función social de la universidad es la generación de conocimiento y su transmisión para la formación de profesionales que atiendan los problemas centrales de la sociedad.

Las organizaciones, por su lado, son la concreción, siempre local y diversa, del mundo institucional. Su primera vinculación con dicho ámbito se puede encontrar en su misión social. Para alcanzar sus resultados, las organizaciones deben generar los medios pertinentes, y son los modos de gestión los esquemas más generales de actuación. En este ámbito la eficiencia adquiere un sentido social. Para comprender los modos de gestión, que son las modalidades operativas generales para llevar a cabo la acción social referida institucionalmente, resulta preciso con- 
siderar las expresiones cualitativas de largo plazo y reconocer las contradicciones institucionales representadas por los diversos participantes (stakeholders) en la organización. El sobredimensionamiento de la eficiencia es una característica de los nuevos modos de gestión, que además enfatizan la rentabilidad de corto plazo y establecen indicadores cuantitativos que se distancian cada vez más de la dinámica organizacional. La noción de responsabilidad social se desdibuja al perderse el sentido institucional de las organizaciones; en este contexto, por ejemplo, los hospitales deben ser rentables y, si no, por lo menos, eficientes: el corto plazo y la cuantificación se instalan en discursos seductores, pero huecos, concretados en esquemas de solidaridad abstractos, que obedecen a mandatos genéricos de urgencia, totalidad y excelencia.

\section{La gran empresa como institución}

La revolución industrial, generada en la segunda mitad del siglo xviri en Gran Bretaña, trajo como una de sus principales consecuencias el desarrollo de la fábrica. Asombrados por los espectaculares desarrollos tecnológicos de entonces, los economistas consideraron que una gran época de desarrollo se iba a iniciar. Frente a las maravillas que prometía la modernidad, no alcanzaron a preguntarse acerca de la responsabilidad social de la fábrica. La tecnología y la división detallada del trabajo permitían lograr niveles nunca antes conocidos de productividad y desarrollo social. Aunado a ello, el mercado era considerado como el dispositivo más adecuado de regulación de las relaciones sociales, al estar sustentado en el equilibrio proveniente del encuentro entre oferta y demanda, cuyo ingrediente principal era una racionalidad sustentada en el egoísmo (Smith, 1776). El mercado dejó de ser solo un espacio físico de intercambio de bienes para constituirse principalmente en un dispositivo de regulación social mediante el establecimiento de precios (Polanyi, 2009); la discusión sobre la responsabilidad social de la fábrica carecía de relevancia al estar ya incorporada, como automatismo, en la capacidad autorreguladora del mercado ${ }^{4}$.

La modernidad surgida de la primera revolución industrial y de las nuevas formas de organización fabril cambió rápidamente su centro de gravedad del Reino Unido hacia los Estados Unidos. El triunfo del norte industrial sobre el sur agrícola en la guerra de Secesión en 1865 propició el desarrollo de grandes empresas. Fue así como surgió, de acuerdo con Chandler (1977), una nueva forma de organización: la corporación. De esta manera, a mediados del siglo xix

\footnotetext{
4 No ignoramos las consecuencias negativas que acarreó la fábrica y que han sido motivo de diversos análisis críticos hasta la fecha. Lo que queremos destacar es el énfasis puesto, tanto por economistas como por empresarios, en la idea general de progreso en detrimento de una conciencia acerca de sus aspectos negativos o bien, en todo caso, cómo estos podrían ser relativamente subsanados (Montaño: 2011).
} 
aparecieron dos grandes empresas de comunicaciones: la de telégrafos y la de los ferrocarriles (Chandler, 1990).

La aparición de la corporación, de acuerdo con el célebre estudio de Berle y Means, no solo acarreó cambios significativos en el interior de la gran empresa, sino que suscitó un debate acerca de su lugar en la sociedad. La transformación más importante, de acuerdo con estos autores, es que las grandes empresas estadounidenses separaban la propiedad del control, la propiedad representada por un amplio número disperso de accionistas, y el control, por un grupo social en ascenso, ingenieros, abogados y administradores, conocidos genéricamente como managers ${ }^{5}$. La empresa familiar, con su orientación paternalista, parecía dejar abruptamente su lugar a grupos de profesionales racionales, especialistas en la toma de decisiones. De hecho, al representar intereses distintos, se planteó incluso un nuevo giro del capitalismo, puesto que la rentabilidad de las acciones no constituía ya el principal objetivo, sino el crecimiento y la diversificación. De acuerdo con Acquier y Aggeri (2008), la fábrica cedió su lugar a la corporación, inaugurando una nueva institución. No obstante, es importante señalar que, de acuerdo con un estudio realizado por La Porta et alii (1999), se observa que no en todos los países se ha dado la denominada revolución de los mánager. Es cierto que dicha transformación ocurre en los países más industrializados, como Reino Unido, Japón y Estados Unidos. En países de desarrollo económico intermedio, como España y Suiza, la propiedad - y, por lo tanto, el control de las grandes corporaciones - se distribuye entre diversos actores, como accionistas, familias y el Estado. En los países latinoamericanos, como Argentina y México, incluidos en dicho estudio, la propiedad se concentra ampliamente en manos de importantes familias (Gráfico 1).

\footnotetext{
5 El término manager ha sido traducido de maneras diversas al castellano, principalmente como administrador, director e incluso gerente; en un principio hacía alusión a los puestos directivos provenientes de diversas formaciones como la economía, el derecho, la ingeniería o la administración. Con el paso del tiempo y el desarrollo de las escuelas de negocios, se fue concentrando cada vez más en el de administrador. El concepto francés de cadre presenta algunos matices ya que este incluye no solo distintas opciones disciplinarias, sino que además hace referencia a niveles jerárquicos también más amplios (Boltanski: 1982). En este sentido, en castellano la palabra administrador hace también en ocasiones referencia a un trabajo de apoyo, de nivel jerárquico intermedio, y no necesariamente de dirección.
} 
Gráfico 1. Propiedad y control. Países seleccionados

Argentina
Australia
Austria
Bégica
Canadá
Corea del Sur
Dinamarca
España
Estados Unidos
Finlandia
Francia
Grecia
Holanda
Hong Kong
Irlanda
Israel
Italia
Japón
México
Noruega
Nueva Zelanda
Portugal
Reino Unido
Singapur
Suecia
Suiza

Fuente: elaborado con base en La Porta et alii, 1999.

Berle y Means constatan que, antes de la separación entre propiedad y control, existió un amplio movimiento de concentración económica en la que intervinieron empresas industriales y de servicios con grandes bancos y otras organizaciones financieras (véanse también los trabajos de Roe, 1994 y Mizruchi, 2004). En este contexto, los autores no se muestran muy optimistas, ya que el paternalismo y las creencias religiosas serían relegadas a un segundo plano por los administradores, quienes se encontrarían más interesados en el crecimiento de la corporación que en la repartición de dividendos o en el bienestar social. Sin embargo, como veremos más adelante, la relativización de la importancia de la ganancia por parte de la corporación, debido a la menor presencia de los accionistas en la toma de decisiones, se conjugó de manera muy adecuada con los principios calvinistas de la ética protestante, y logró establecer un alto nivel de congruencia entre ambas instituciones. 


\section{La ética protestante y el espíritu del capitalismo managerial}

La corporación, en cuanto institución, no funciona de manera aislada a su mundo social y no se reduce, por lo tanto, a la sola presencia de la racionalidad, sino que se sustenta ampliamente en la religión protestante. Como es bien conocido, los trabajos de Weber (1991) establecieron una relación, aunque temporal, entre ambos tipos de institución.

Para empezar, podemos señalar que la tradición, siguiendo los planteamientos de Weber, se caracteriza por la centralidad de una institución, la iglesia; esta proporcionaba sentido a otras instituciones, como el ejército, la familia, la escuela, el hospital e incluso aquellas dedicadas a las artes. El gran centro generador de sentido y responsabilidad social lo constituía la iglesia. En esta misma dirección, como sabemos, Weber señala que el capitalismo se desarrolló gracias al impulso de los valores y principios de la ética protestante. La modernidad puede ser interpretada, en este marco, como una creciente autonomía institucional impulsada, entre otros, por el gran desarrollo de la racionalidad instrumental (Weber, 1970). Esta convivencia institucional entre la iglesia y la empresa tuvo, empero, una corta duración; rápidamente la ética protestante dejó su lugar a los principios y valores de la gran corporación, tal como en su momento expresó el autor nostálgicamente: «El espíritu se esfumół el cofre permanece vacío, sin que pueda saberse si para siempre. Como quiera que sea, el capitalismo triunfante, siendo que se apoya en bases mecánicas, ya no requiere más de la ayuda religiosa» (Weber, 1991: 112).

Pero, ¿hasta dónde han desaparecido los valores religiosos realmente de las sociedades económicamente más desarrolladas? De acuerdo con las primeras visiones antropológicas, la tradición no desaparece, sino que tiende a entremezclarse con la modernidad. Tylor (2009) y Durkheim (2000) son dos excelentes ejemplos de esta orientación: permiten pensar que la tradición y la modernidad no son mutuamente excluyentes, como generalmente se piensa, sino que son más bien mutuamente complementarias (Montaño, 2004). De esta manera, algunas de las primeras manifestaciones de la responsabilidad social de las grandes em-

presas que se empezaron a plantear en la década de los veinte del siglo pasado en los Estados Unidos tuvo una fuerte carga religiosa. La creación de la corporación, constituida por un alto número de accionistas, hacía crecer un fuerte temor acerca del papel social de la gran empresa; entonces la invocación religiosa no se hizo esperar, tal como demuestra un artículo publicado a finales de la década de los treinta del siglo pasado en el que se señala que solo mediante la cooperación entre los diferentes actores que interactúan con la empresa (trabajadores, adminis- 
tradores, accionistas y clientes) podría dársele un sentido social a su desarrollo: «Entonces, y solo entonces, podrá la máquina ser puesta bajo el completo control del hombre y ser utilizada exclusivamente para aquellos propósitos que tienen sus raíces en los más finos impulsos de la mente y el espíritu -impulsos en los que participa Dios, el creador y soberano de todo» (Young, 1939: 92).

Existe un acuerdo muy generalizado en reconocer que la primer gran obra escrita sobre el tema de la Responsabilidad Social Corporativa - Social Responsibilities of the Businessman - fue realizada por Howard Bowen en 1953 (Acquier y Gond, 2007; Caroll, 1999). Dicho texto forma parte de un proyecto promovido por una organización religiosa, Federal Council of the Churches of Christ in America, para elaborar una serie de planteamientos que orientaran la dirección de las empresas, tomando como fuente de inspiración el espíritu protestante y los planteamientos centrales de la célebre encíclica Rerum Novarum, expedida por el Papa León XIII en 1891. Bowen se preguntaba por qué existía en su época un mayor interés por las cuestiones de la responsabilidad que en años previos. $\mathrm{Su}$ respuesta consta de tres argumentos. El primero indica que la causa era que los hombres de negocios se encontraban obligados, presionados por diversos grupos, como los sindicatos y el Estado, a considerar aspectos en los que habían sido fuertemente criticados. El segundo indica que era debido a que existía un mayor nivel de persuasión; la constante comunicación con otros actores sociales y el peso creciente de las escuelas de administración proponían, señala el autor, considerar a la empresa en su entorno social. Finalmente, el tercer argumento encontraba las raíces de esta cuestión, precisamente, en la separación entre la propiedad y el control, en la idea de que la corporación era más que una entidad en busca de beneficios económicos para los accionistas; estos ya no eran el único grupo interesado en el desempeño de la empresa. Los administradores guardaban un alto nivel de autonomía con respecto a los accionistas y podían, por lo tanto, responder de manera más adecuada a los intereses de los trabajadores, consumidores, gobierno, etc.; se supone que el horizonte social de los mánager era amplio, además de ser considerados personas preparadas, con conocimientos profesionales y capaces de atender los problemas que la operación de estos sistemas supone.

Bowen dedica un capítulo a la visión protestante de la RSC, que extrae de una serie de entrevistas con hombres de negocios y con académicos. Concluye que, desde el punto de vista religioso, es necesario transitar hacia un «sistema mixto» en el que se logre combinar armónicamente aspectos tanto económicos como sociales. Así, considera que dicho sistema debe contener «elementos de la empresa privada, la cooperativa y la pública, debe estar motivado parcialmente 
por intereses particulares y sociales, predominando estos segundos; controlado parcialmente por la competencia tanto como por el interés social y la regulación pública y distribuir el ingreso tanto con base en los precios de mercado como en función de las necesidades de las personas» (Bowen, 1953: 39).

De acuerdo con Pasquero (2005), los grupos religiosos han sido siempre muy influyentes en el seno de la gran empresa estadounidense. El autor refiere que una de sus tácticas consiste en adquirir acciones con el fin de participar en las asambleas de accionistas y exponer públicamente su visión sobre diversos hechos económicos, sociales y políticos. En efecto, al contrario de lo que se piensa generalmente, los países industrializados aún conservan arraigadas sus creencias religiosas. En este sentido, como constata el trabajo de Ingleheart y Carballo (2008), un poco más de la mitad de la sociedad estadounidense mantiene vivas sus creencias religiosas y resulta además ser una de las sociedades más tradicionales del mundo económicamente desarrollado.

\section{El saber experto: las escuelas de negocios}

Las primeras escuelas de negocios — business schools - en Estados Unidos fueron fundadas hacia finales del siglo xix, la primera de ellas se instaló en la Universidad de Pensylvania en 1881. En 1910 se estimaba que el número había crecido a 14 y en 1925 había más de 180 (Acquier y Aggeri, 2008). Si bien en la actualidad muchas de estas escuelas gozan de alto prestigio, es importante señalar que recién terminada la Segunda Guerra Mundial la situación era totalmente distinta: «En general, los programas de negocios eran considerados como entre los más pobres en sus respectivos campus. Sus estudiantes habían tenido los más bajos promedios, sus profesores generalmente habían sido reclutados entre oficiales militares retirados y ejecutivos de negocios que no tenían doctorado; y tanto la pedagogía como la investigación tendían a no ser teóricas y orientadas hacia la práctica» (Cotton et alii, 2001: 227).

La Association to Advance Collegiate Schools of Business (AACSB) se fundó en 1916 con el fin de impulsar, mediante la acreditación, la calidad de las escuelas de negocios, y es considerada en la actualidad como la acreditación más prestigiosa en el mundo académico en el ámbito de la gestión de empresas. En un inicio solo tenían acceso a ella las escuelas estadounidenses, y no fue hasta 1997 que se aceptó a la escuela francesa ESSEC (École Supérieure des Sciences Économiques et Commerciales) en sus filas. Para finales del 2012, ya habían sido incorporadas unas 650 escuelas de cerca de 50 países, convirtiéndose en un dispositivo legitimador al ser considerado un símbolo internacional de calidad. Actualmente, promueve entre sus agremiados la importancia de la ética en el 
proceso educativo y enfatiza su incorporación en los currículos de los cursos. Establece también estándares con los que deben cumplir las escuelas de negocios, como códigos de conducta, sistema de valores, códigos de honor, procedimientos para atender malas conductas, entre otros. Entre sus principios con respecto a la responsabilidad social destaca, de acuerdo con su portal web, el desarrollo de «las capacidades de los estudiantes para que sean futuros generadores del valor sustentable para las empresas y la sociedad y para trabajar por una economía global incluyente y sustentable».

La gran corporación, los mánager y las escuelas de negocios son producto de un mismo desarrollo histórico. El denominador común es la búsqueda de la eficiencia, entendida como la relación entre resultados e insumos. En el caso de los mánager, el énfasis se ha colocado históricamente en la productividad, mientras que para el grupo de accionistas este ha recaído en la rentabilidad. De hecho, es la eficiencia productiva la piedra de toque de la administración como disciplina, entendida esta, desde la propuesta taylorista, como una manera de resolver el conflicto entre patrones y obreros. Taylor comienza su famosa obra, Principios de la Administración Científica estableciendo claramente su intención: «El objeto principal de la administración ha de ser asegurar la máxima prosperidad para el patrón, junto con la máxima prosperidad para cada uno de los empleados» (Taylor, 1961: 19). El medio es la productividad; este, sin un fin adecuado, carecería de sentido, tal como se señala en los inicios mismos de la disciplina administrativa; las escuelas de negocios deben considerar no solo la productividad, sino su sentido social: «Es el fin hacia el cual se dirige la eficiencia lo que se cuestiona. La crítica principal no es contra el contenido de la currícula. Es el espíritu y el punto de vista lo que hará o estropeará el trabajo desde la perspectiva del bien público» (Wooster, 1919: 60). Es importante también recordar el apoyo que las universidades, y no solo las escuelas de negocios, recibieron de importantes empresarios, como Carnegie, Mellon y Sloan, entre otros (Knights y Morgan, 1991), así como el apoyo del taylorismo para mejorar su eficiencia (Callahan, 1962).

En el ámbito de la elaboración teórica, resulta interesante señalar que, desde la perspectiva sociológica, la gran empresa se convierte, desde el funcionalismo estructural de Parsons, en un engranaje más del gran sistema social y debe estar al servicio de este y no solo de sus propietarios: «Para la empresa privada, el beneficio económico es una medida básica y un símbolo de éxito, y forma por lo tanto parte de la estructura de objetivos de la organización. Pero no puede ser el objetivo primario ya que la utilidad económica no constituye por sí misma una función en nombre de la sociedad en tanto sistema» (Parsons, 1956: 68). La productividad no es solo un problema técnico ni se inscribe solo en el ámbito 
económico; la administración tiene la ardua tarea de reelaborar este dispositivo para darle un mayor significado social, que no debe estar solo al servicio de unos cuantos, sino de una amplia variedad de actores. El administrador, desde esta óptica, se convierte así en un personaje que asume una alta responsabilidad social, y esa es la tarea de las escuelas universitarias de negocios, tal como expresara Elton Mayo en 1933 al finalizar su primer libro, Problemas humanos de una civilización industrial: "No tenemos suficientes administradores que se den cuenta de que el problema que se les plantea no es económico, sino humano y social. Las universidades del mundo están admirablemente equipadas para el descubrimiento y la formación de científicos especializados; pero todavía no han empezado a pensar en el descubrimiento ni en la formación del administrador moderno» (Mayo, 1972: 170).

Por otro lado, Chester Barnard ha sido sin duda uno de los escritores más influyentes del pensamiento managerial. En su conocido libro, Las funciones del ejecutivo, publicado por primera vez en 1938, introdujo diversos aspectos que orientaron el desarrollo tanto de la disciplina administrativa como de la teoría de la organización. Uno de estos aspectos es precisamente el de la responsabilidad. El autor postula que cada individuo posee «códigos privados morales», producto tanto de los diversos entornos en los que participa como de las trayectorias personales, y define la responsabilidad como «el poder de un código moral particular para controlar la conducta del individuo en la presencia de fuertes deseos o impulsos contrarios» (Barnard, 1971: 263). La responsabilidad se vuelve un fenómeno complejo cuando se reconoce, señala Barnard, que los individuos poseemos diversos códigos, provenientes de esferas de acción distintas, como la familia, la iglesia, la empresa, el gobierno, etc. Estos códigos son portadores de valores y pueden entrar en contradicción unos con otros en función de cuál es su fuente, y generar conflictos inevitables. La función principal del directivo es, pues, resolver dichos dilemas ya sea tratándolos técnicamente como si fueran problemas, o bien generando nuevos códigos morales. Retomando algunos planteamientos centrales de la propuesta de Barnard, Herbert Simon (1982), plantea la idea del equilibrio organizacional como condición de armonía social, este concepto se encuentra en la base de la teoría de los grupos participantes (stakeholders) como fundamento del comportamiento de los mánager y su noción de responsabilidad social (Kakabadse et alii, 2005). De acuerdo con Simon, todos los participantes - trabajadores, accionistas, proveedores, consumidores, etc.- tienen interés en permanecer vinculados a la organización en tanto que sus retribuciones sean mayores o iguales a sus contribuciones. En el caso de que todos los miembros 
interesados logren dicho estado, la organización habrá alcanzado su estado de equilibrio; la eficiencia juega un papel primordial en dicho proceso.

La crisis económica que atravesó Estados Unidos en los setenta - baja de productividad, crecimiento de desempleo, pérdida de competitividad, crisis energética, etc. - acarreó cambios significativos. Diversas voces críticas, principalmente desde el nuevo institucionalismo económico, cuestionaron ampliamente el papel de los mánager; teorías como la de los costos de transacción, de la agencia y de los mercados financieros pusieron en tela de juicio la capacidad de los mánager como agentes de la regulación social, y surgió nuevamente la idea de que el mercado constituiría la mejor opción de organización social (Khurana, 2007). Aunado a ello, el gran auge de los programas de maestría en administración motivó que las escuelas de negocios entraran de lleno en los mercados internacionales. Debido a la creciente importancia y dinamismo de los mercados financieros desregulados, de mayor acceso gracias a los nuevos dispositivos de información y comunicación, los programas generales de administración incorporaron cada vez más la enseñanza de las finanzas. En este ámbito, muchos programas de investigación, con el apoyo de modelos matemáticos, han conocido un gran auge y se les ha otorgado una imagen de seriedad en la investigación científica.

Conforme avanzaba el modelo financiero promovido por economistas y accionistas, se empezaron a suscitar algunos escándalos corporativos, influidos por el nuevo modelo de desarrollo; entre los más importantes de la década de los noventa del siglo pasado se pueden señalar, de acuerdo con Jennings (1999), los siguientes: Archer Daniels Midland, Long-Term Capital, Cendant, Banker Trust, Rite-Aid, WalMart, Sears, Enron, WorldCom, Sunbeam, Arthur Andersen y Health South. Algunos de ellos conocieron una amplia difusión, como es el caso de Enron, que había desarrollado incluso una serie de dispositivos administrativos para asegurar la RSC, por ejemplo, un sistema de valores y códigos de ética. En dicho escándalo se vieron además involucradas la famosa Harvard Business School, la escuela de negocios más reputada por su MBA, así como una de las empresas consultoras más connotadas a nivel internacional, la Arthur Andersen (Alsop, 2006). Estos escándalos implicaron no solo una llamada de atención con respecto a los efectos perversos que acarreaban los nuevos modos de gestión sustentados en la preeminencia financiera, sino también una fuerte crítica a la visión y la eficacia de los instrumentos éticos promovidos por las escuelas de negocios. Si bien el esquema de los MBA se resintió de los efectos de estos escándalos y de la crítica académica (Mintzberg, 2004), estos programas salieron adelante.

De esta manera, las principales escuelas de negocios continúan impartiendo en sus programas de maestría cursos de ética, responsabilidad social corpora- 
tiva y desarrollo sustentable (Christensen et alii, 2007) y siguen atendiendo de manera escrupulosa los criterios estandarizados de la AACSB (McCraw et alii., 2009). Los programas de maestría en administración cuentan en la mayoría de los países con un gran reconocimiento, a pesar de las críticas que han recibido en años recientes. Estos programas son altamente demandados por el impulso a las trayectorias profesionales personales; entre los más reconocidos internacionalmente destacan, de acuerdo con el Financial Times, las maestrías estadounidenses (16 entre las primeras 20), seguidas de las europeas (6), las hindúes (2) y las chinas (1). Por otro lado, hay que señalar que los programas de maestría en finanzas se han incrementado en años recientes; así, esta lista los incluye a partir de 2006. Estos no han alcanzado todavía el prestigio de las maestrías en administración; los programas de finanzas se han desarrollado principalmente en Europa (18 de los 20 primeros), solo uno de ellos es estadounidense y otro chino. La diferencia de los salarios de los egresados una vez incorporados al mercado de trabajo es muy marcada, más del doble para los programas de administración aunque, en promedio, no existe diferencia en cuanto a la empleabilidad: más del $90 \%$ en ambos casos se encuentra trabajando en los primeros tres meses siguientes a la conclusión de los estudios (Tabla 1).

Si bien la revolución de los mánager y el concomitante desarrollo de las escuelas de negocios son fenómenos sociales que ocurren principalmente en Estados Unidos, sus efectos se despliegan a escala internacional. De esta manera, las escuelas de negocios en la mayor parte del mundo han integrado las preocupaciones éticas de la responsabilidad social en su currículos, como acontece, entre otros muchos espacios, en el mundo iberoamericano (Licha, 2012; Martínez y García, 2012; Naciones Unidas, 2010). 
Tabla 1. Principales programas de maestría en administración y en finanzas. País de origen, salario medio y empleo (en \%)

\begin{tabular}{|c|c|c|c|c|}
\hline & Maestrias en administracion de negocios & País & $\begin{array}{c}\text { Salario } \\
\text { (dólares) }\end{array}$ & $\begin{array}{l}\text { Empleo, } \\
\text { en } \%\end{array}$ \\
\hline 1 & Stanford Graduate School of Business & EU & 192,179 & 92 \\
\hline 2 & Harvard Business School & EU & 178,249 & 93 \\
\hline 3 & University of Pennsylvania: Wharton & EU & 172,353 & 85 \\
\hline 4 & London Business School & RU & 152,981 & 85 \\
\hline 5 & Columbia Business School & EU & 166,497 & 91 \\
\hline 6 & Insead & FRA/SIN & 144,355 & 84 \\
\hline 7 & MIT: Sloan & EU & 157,337 & 92 \\
\hline 8 & IE Business School & ES & 156,658 & 92 \\
\hline 9 & Iese Business School & ES & 133,888 & 97 \\
\hline 10 & Hong Kong UST Business School & $\mathrm{CHI}$ & 127,600 & 89 \\
\hline 11 & $\begin{array}{l}\text { Indian Institute of Management, } \\
\text { Ahmedabad }\end{array}$ & IND & 175,076 & 96 \\
\hline 12 & University of Chicago: Booth & EU & 152,585 & 89 \\
\hline 13 & IMD & SUI & 144,045 & 91 \\
\hline 14 & University of California at Berkeley: Haas & EU & 146,811 & 91 \\
\hline 15 & Duke University: Fuqua & EU & 139,405 & 91 \\
\hline 16 & Northwestern Universityः Kellogg & EU & 145,811 & 91 \\
\hline 17 & New York University: Stern & EU & 134,093 & 91 \\
\hline 18 & HEC Paris & FRA & 121,061 & 90 \\
\hline 19 & Dartmouth College: Tuck & EU & 151,182 & 94 \\
\hline \multirow[t]{3}{*}{20} & Indian School of Business & IND & 129,512 & 100 \\
\hline & Promedio & & 151,084 & 91 \\
\hline & Maestrías en finanzas & & & \\
\hline 1 & HEC Paris & FR & 93,680 & 96 \\
\hline 2 & Essec Business School & FRA/SIN & 74,249 & 92 \\
\hline 3 & IE Business School & ESP & 91,262 & 94 \\
\hline 4 & University of Oxford: Saïd & RU & 91,213 & 98 \\
\hline 5 & University of St Gallen & SUI & 77,703 & 100 \\
\hline 6 & Edhec Business School & FRA & 58,786 & 89 \\
\hline 7 & ESCP Europe & $\begin{array}{c}\text { FRA/RU/ } \\
\text { ALE/ESP/ } \\
\text { ITA }\end{array}$ & 65,062 & 94 \\
\hline 8 & Peking Universityः Guanghua & $\mathrm{CHI}$ & 96,802 & 100 \\
\hline 9 & Grenoble Graduate School of Business & FRA & 56,576 & 92 \\
\hline 10 & Warwick Business School & RU & 56,576 & 92 \\
\hline 11 & Cranfield School of Management & RU & 79,612 & 81 \\
\hline 12 & Skema Business School & FRA & 55,878 & 93 \\
\hline
\end{tabular}




\begin{tabular}{|c|l|c|c|c|}
\hline 13 & Imperial College Business School & RU & 55,878 & 93 \\
\hline 14 & HEC Lausanne & SUI & 65,728 & 100 \\
\hline 15 & City University: Cass & RU & 62,258 & 83 \\
\hline 16 & EM Lyon Business School & FRA & 57,590 & 85 \\
\hline 17 & Brandeis University & EU & 69,812 & 93 \\
\hline 18 & Stockholm School of Economics & SUE & 67,812 & 93 \\
\hline 19 & $\begin{array}{l}\text { Rotterdam School of Management, } \\
\text { Erasmus University }\end{array}$ & HOL & 59,680 & 92 \\
\hline 20 & Eada & ESP & 59,993 & 82 \\
\hline & Promedio & 69,808 & 92 \\
\hline
\end{tabular}

Nota: EU: Estados Unidos. RU: Reino Unido. FRA: Francia. SIN: Singapur. ESPः España. CHI: China. IND: India. SUI: Suiza. ALEः Alemania. ITA: Italia. SUE: Suecia. HOL: Holanda. El salario es anualizado. El empleo hace referencia al porcentaje de egresados que tienen trabajo en un período no mayor a tres meses.

Fuente: elaborado con base en Financial Times: 2013.

\section{De la productividad a la rentabilidad}

Henri Fayol y Henry Ford constituyen dos personajes claves que pueden ser considerados como prototípicos del administrador profesional y, por lo tanto, de la sociedad managerial. El primero trabajó como director general de la empresa Société Commentry-Fourchambault et Decazeville, y destacó que la función principal del director es atender el interés social. Es importante señalar que este personaje no guardaba relación familiar alguna con los propietarios ni poseía acciones de la empresa. En esta época, los dueños de las minas se apoyaban ampliamente en la iglesia, brindando generosos apoyos a diversas actividades y era costumbre solicitar la fe de bautizo en el momento de contratar a los empleados. Fayol se mostraba respetuoso frente a estas tradiciones y señalaba que la organización industrial había generado tan buenos resultados porque esta se había apoyado en la organización militar (Cohen, 2013). Esta vinculación fue muy evidente en Estados Unidos al final de la Segunda Guerra Mundial.

Por su lado, Ford representa una figura todavía mucho más emblemática que la de Fayol. Es bien conocida su célebre decisión de incrementar los salarios de 2,3 a 5 dólares al día con la idea de propiciar una mayor demanda para hacer frente al crecimiento continuo de la producción, pero también como una forma para disminuir la alta tasa de rotación de los obreros debido al descontento frente a la cadena de montaje (Coriat, 1993; Friedmann, 1977). Su política paternalista, que incluía una extrema vigilancia del comportamiento de los obreros fuera de la fábrica, fue duramente criticada (Gramcsi, 2000). Su participación activa en la industria militar en la Segunda Guerra Mundial le granjeó muchas simpatías 
y la búsqueda de la productividad le llevó a desarrollar ampliamente la noción de estandarización, que posteriormente se encontró en diversos programas de certificación ISO, incluida la reciente versión 26000 , aplicable a programas de RSC (Rendón, 2012; Quairel-Lanoizelée, 2011). Ford era el principal accionista de su empresa, seguido por los hermanos Dodge. En 1916 los Dodge pretendieron retirarse de la empresa y, con los dividendos, montar su propia empresa automotriz. Sin embargo, Ford decidió no repartir dividendos en ese momento para poder invertir en su empresa y lograr precios más bajos para sus vehículos, ya que estaba convencido de que la maximización del beneficio económico no era lo más importante en la venta de coches. Los hermanos Dodge apelaron dicha decisión en la corte y vencieron, lo que forzó a Ford a distribuir los dividendos correspondientes, el juez le recordó que la principal obligación de una empresa es con sus inversionistas. (Mullerat, 2007).

El período posterior a la Segunda Guerra Mundial mejoró la imagen de los administradores, entre ellos, evidentemente la de Ford, que se había perdido durante la gran depresión económica (1929-1933). Muchos de los logros que se obtuvieron en la industria militar se trasladaron, en forma de modelos y discursos, al terreno industrial. Sin embargo, la idea de una sociedad dirigida por administradores profesionales poco a poco fue perdiendo prestigio. De hecho, se trata de la gran decepción frente a los planteamientos de un antiguo troskista, James Burnham (1941), quien había vaticinado una «sociedad managerial», en la que la propiedad privada desaparecería y dejaría la conducción de los negocios a un grupo selecto de profesionales de alto nivel. Sin embargo, instalada la Guerra Fría, la responsabilidad social de los directores se asumió como la defensa del capitalismo frente a la amenaza roja: «La guerra fría también acarreó expectativas del nivel de responsabilidad social, dado que los directores castigaban a los presuntos comunistas, reportaban amigos, familiares y vecinos subversivos y emprendían campañas para convencer a los americanos que apoyar a los sindicatos significaba apoyar el estado socialista» (Genoe, 2011: 420).

Una organización empresarial surgió en plena Guerra Mundial, el Committee for Economic Development (CED), fundada en 1942 con el objeto de contribuir a estabilizar la economía estadounidense una vez terminado el conflicto bélico. Esta organización está constituida por directores de grandes empresas y presidentes de universidades. Goza de un gran prestigio en temas económicos, políticos y sociales y sus propuestas han propiciado la creación de instituciones financieras tan importantes como el Fondo Monetario Internacional y el Banco Mundial (Pasquero, 2005). Entre sus programas actuales se encuentra uno orientado hacia la gobernanza corporativa (CED, 2009). El CED ha reconocido 
la creciente importancia de los inversionistas en el contexto de la separación de la propiedad y el control, y ha manifestado una triple preocupación: la primera consiste en recuperar la confianza del público después de los grandes escándalos financieros recientes; la segunda se refiere a la necesidad de promover el desarrollo empresarial a largo plazo, haciendo frente a la exigencia de rentabilidad a corto plazo, propia de los mercados financieros actuales; la tercera se orienta hacia la reconstrucción del liderazgo de la gran empresa mediante la convergencia del desempeño de largo plazo con los objetivos de la sociedad.

Los últimos años de la década de los setenta del siglo pasado representan un hito en la historia reciente. Fenómenos de tipo económico, social, político, tecnológico y cultural bosquejaron una nueva realidad, conceptualizada de maneras distintas - posmoderna, hipermoderna, supermoderna, moderna acelerada, etc.- que pusieron el acento en la crisis de las instituciones sociales en tanto que dispositivos promotores de valores y comportamientos (Aubert, 2004; Dubet, 2002). Nuevas formas de organización, conocidas generalmente como posburocráticas, posmodernas, flexibles, de clase mundial etc., se vieron acompañadas de nuevos modelos de gestión que acentuaron la ganancia económica, el corto plazo, las estrategias de globalización, el uso intensivo de tecnologías y la utilización del indicador cuantitativo como único representante de la realidad.

El neoliberalismo resultó, como su nombre indica, un concepto con doble significado. Por una parte, se trata de un regreso a la idea de que el mercado constituye el mecanismo regulador por excelencia de las relaciones sociales $y$, por la otra, hace referencia a un nuevo fenómeno con características inéditas dada su ocurrencia en un contexto de modernidad exacerbada. El neoliberalismo representa entonces un fenómeno que implica el desarrollo de un nuevo esquema de regulación social, en el que se constata la pérdida relativa de poder de los administradores profesionales en beneficio de grandes inversionistas. Los problemas económicos - bajo crecimiento económico, inflación, deuda, baja productividad, incremento de la competencia internacional- que sufrieron los Estados Unidos a finales de la segunda mitad de la década de los setenta comenzaron a cuestionar la capacidad de los administradores de las grandes empresas para conservar el equilibrio social. Algunos esfuerzos por recuperar dicha preeminencia en la sociedad fueron precisamente las nuevas formas de organización, que no solo han provocado despidos masivos, suicidios en el trabajo y violencia psicológica, entre otros, sino que han inundado a la sociedad en su conjunto de un herramental conceptual e instrumental que ha trastocado la vida social de los individuos, promoviendo diversas crisis institucionales (Gaulejac, 2005). La figura del mercado retoma un nuevo aliento bajo los auspicios de una organización empresarial 
poderosa, la Business Roundtable, y de la teoría económica de Hayeck (2007) y de Friedman (1966; 1970): el mundo social deja en parte de existir para dar pie a una racionalidad oportunista con referencias abstractas. Se constata una tensión creciente entre los intereses de los accionistas y los de los administradores en este cambio de esquema de regulación social. Así, Aglietta, desde la corriente francesa de la economía de la regulación, ahonda en las modalidades del nuevo capitalismo, enfatizando la actual tensión creada por la incorporación de los grandes inversionistas: «En el nuevo régimen de crecimiento, la variable central del comportamiento de las empresas es la utilidad por acción. La gobernanza de los accionistas institucionales fuerza a las empresas a maximizar esta variable. El criterio de desempeño actúa sobre las decisiones estratégicas de las empresas, las estructuras de decisión, el reparto y la utilización de las utilidades. De ello resulta una tensión entre la distribución de dividendos a los accionistas y la utilidad retenida por los administradores...» (Aglietta, 1999: 279).

\section{Tres reflexiones finales}

Para finalizar, quisiéramos señalar tres aspectos que consideramos relevantes en esta reflexión. El primero de ellos es que, como hemos podido observar a lo largo de este trabajo, el tema de la RSC resulta altamente complejo, tanto desde el punto de vista de la acción social como de su comprensión teórica. Se han destacado algunos actores institucionales que es necesario considerar para lograr entender las vicisitudes que ha recorrido el proceso de institucionalización de la RSC. Algunos otros, sin embargo, dadas las limitaciones de espacio, han quedado por el momento fuera del análisis, o han sido mencionados tangencialmente; queda pendiente incorporarlos o trabajarlos con mayor detenimiento, entre ellos, rescatamos los siguientes: el Estado y sus instancias reguladoras, las organizaciones internacionales, las asociaciones acreditadoras, los grupos de consumidores, los sindicatos y las centrales obreras, las organizaciones no gubernamentales, las empresas de consultoría administrativa y las empresas editoriales especializadas en temas de gestión.

Una segunda reflexión lleva a reconocer que, si bien el origen y la evolución del fenómeno de la RSC se encuentra fuertemente anclado en un espacio y tiempo particulares, sus resultados se observan, empero, a nivel global. La transferencia de discursos y modos de organización y gestión no pueden verse solo como una moda administrativa ni como el avance de un rampante isomorfismo organizacional. La descontextualización de modelos y programas aporta elementos de respuesta que, aunados a la simplicidad discursiva que relaciona de manera abstracta la eficiencia con el desarrollo, permite explicar otra parte del fenómeno. 
A ello debemos agregar la posibilidad de que la aplicación de estos modelos en realidades diversas permite enfrentar problemas distintos con las mismas fórmulas de solución, con la impresión, además, de incrustarse en procesos de modernización ya probados. Lo mismo acontece, desde otro ángulo, en términos de su traslado institucional. El discurso de la RCS, a pesar de que algunos investigadores han reconocido sus múltiples facetas, se viene constituyendo en un programa único, independientemente de su adscripción institucional. En este sentido, resulta paradójico pensar que una de las instituciones socialmente más criticadas se convierta ahora en modelo general, atentando con ello contra la diversidad institucional que requiere toda sociedad; pareciera el inicio de una institucionalización sin instituciones.

Finalmente, la tercera y última reflexión conduce a cuestionar la verdadera utilidad de los programas de RSC, que se suman a la tendencia que han tenido otros instrumentos de gestión y que consiste en formalizarse y pasar a formar parte del inventario de soluciones prêt-à-porter, como códigos de conducta, cursos universitarios, sistemas de indicadores, entre otros, que actúan casi exclusivamente en el plano del discurso legitimador (Boltanski y Chiapello, 2002; Montaño, 2012b) sin transformar esencialmente las prácticas existentes, y sobre todo en la época actual en la que se promocionan mucho los temas de la RSC, tal vez precisamente porque esta ha extraviado su rumbo en el modelo actual imperante. 


\section{Bibliografía}

Abrahamson, Eric (1996), «Management Fashion», en Academy of Management Review, vol. 21, n. ${ }^{\circ} 1$, pp. 254-285.

Acquier, Aurélien y Jean-Pascal Gond (2007), «Aux sources de la responsabilité sociale de l'entreprise: à la (re)découverte d'un ouvrage fondateur, Social Responsibilities of the Businessman d'Howard Bowen», en Finance Contrôle Stratégie, vol. 10, n. ${ }^{\circ}$ 2, pp. 5-35.

Acquier, Aurélien y Franck Aggeri (2008), «Une généalogie de la pensée managériale sur la RSE», en Revue française de gestión, vol. 1, n. ${ }^{\circ}$ 180, pp. 131-157.

Ahme, Góran y Nils Brunsson (2005), «La regulación suave desde una perspectiva organizacional», en Gestión y Política Pública, Centro de Investigación y Docencia Económicas, vol. XIV, n. 3, pp. 527-555.

Aglietta, Michel (1999), «Les transformations du capitalisme contemporain», en Bernard Chavance, Eric Magnin, Ramine Motamed-Nejad y Jacques SAPIR (dirs.), Capitalisme et socialisme en perspective. Evolution et transformation des systèmes économiques, La Découverte, París, pp. 275-292.

Alsop, Ronald J. (2006), «Business Ethics Education in Business Schools: A Commentary», en Journal of Management Education, vol. 30, n. ${ }^{\circ} 1$, pp. 11-14. Aubert, Nicole (2004), «Un individu paradoxal», en Nicole Aubert (dir.), L'individu bypermoderne, Érès, París, pp. 13-24.

Barnard, Chester I. (1971), The Functions of the Executive, Harvard University Press, Cambridge (1938).

Bauman, Zygmunt (2010), Ética posmoderna, Siglo xxi, México (1993).

Berger, Peter L. y Thomas Luckmann (1972), La construcción social de la realidad, Amorrortu, Buenos Aires (1966).

Berle, Adolf y Gardiner Means (1932), The modern corporation and private property, Macmillan, Nueva York.

BolTANSKY, Luc (1982), Les cadres. La formation d'un groupe social, Les éditions de minuit, París.

- y Eve Chiapello (2002), El nuevo espiritu del capitalismo, Akal, Madrid (1999).

Bowen, Howard R. (1953), Social Responsibilities of the Businessman, Harper \& Brothers, Nueva York.

Burnham, James (1941), The Managerial Revolution. What is happening in the world, The John Day Company, Nueva York. 
Callahan, Raymond E. (1962), Education and the Cult of Efficiency. A Study of the Social Forces that have shaped the Administration of Public Schools, The University of Chicago Press, Chicago.

Carroll, Archie (1999), «Corporate Social Responsibility. Evolution of a Definitional Construct», en Business E Society, vol. 38, n.* 3, pp. 268-295.

Castoriadis, Cornelius (1982), La institución imaginaria de la sociedad, Tusquets, Buenos Aires (1975).

- (2006), Figuras de lo pensable, Fondo de Cultura Económica, Buenos Aires (1978).

Chandler, Alfred D. Jr. (1977), The Visible Hand: The Managerial Revolution in American Business, Harvard University Press, Cambridge.

- (1990), Scale and Scope. The Dynamics of Industrial Capitalism, Harvard University Press, Cambridge.

Christensen, Lisa Jones; Ellen Peirce; Laura P. Hartman; W. Michael Hoffman y Jamie Carrier (2007), «Ethics, CSR, and Sustainability Education in the "Financial Times" Top 50 Global Business Schools: Baseline Data and Future Research Directions», en Journal of Business Ethics, vol. 73, n. ${ }^{\circ} 74$, pp. 347-368.

Cohen, Yves (2003), «Fayol, un instituteur de l'ordre industriel», en Entreprises et histoire, n. 34, pp. 29-67.

Committee for Economic Development (2009), Rebuilding Corporate Leadership: How Directors Can Link Long-Term Performance with Public Goals, Washington.

Coriat, Benjamin (1993), El taller y el cronómetro. Ensayo sobre el taylorismo, el fordismo y la producción en masa, Siglo xxi, México (1979).

Cotton, Chester C.; John F. McKenna; Stuart Van Auken y Matthew L. Meuter (2001), «Action and Reaction in the Evolution of Business School Missions», en Management Decision, vol. 39, n.* 3, pp. 227-232.

Czarniawska, Barbara (2005), «Fashion in Organizing», en Barbara Czarniawska y Guje Sevón (eds.), Global ideas. How Ideas, Objects and Practices Travel in the Global Economy, Liber \& Copenhagen Business School Press, Copenhague.

DiMaggio, Paul J. y Walter W. Powell (2001), «Retorno a la jaula de hierro: el isomorfismo institucional y la racionalidad colectiva en los campos organizacionales», en Powell, Walter W. y Paul DiMaggio (comps.), El nuevo institucionalismo en el análisis organizacional, Colegio Nacional de Ciencias Políticas y Administración Pública, Universidad Autónoma del Estado de México y Fondo de Cultura Económica, México, pp.104-125 (1991). 
Dubet, François (2002), Le declin de l'institution, Seuil, París.

Durкheim, Emile (1894), Les règles de la méthode sociologique, página web: $<$ http://classiques.uqac.ca/classiques/Durkheim_emile/regles_methode/ durkheim_regles_methode.pdf $>$.

- (2000), Las formas elementales de la vida religiosa, Colofón, México (1912).

Enriquez, Eugène (1992), L'organisation en analyse, Presses Universitaires de France, París.

Financial Times (2013), Global MBA Ranking, página webः < http*//rankings. $\mathrm{ft}$. com/businessschoolrankings/global-mba-ranking-2013>.

Friedland, Roger y Robert R. Alford (2001), «Introduciendo de nuevo a la sociedad: símbolos, prácticas y contradicciones institucionales», en Walter W. Powell and Paul J. DiMaggio (comps.), El nuevo institucionalismo en el análisis organizacional, Fondo de Cultura Económica, Colegio Nacional de Ciencias Políticas y Administración Pública and Universidad Autónoma del Estado de México, Mexico, pp. 294-329 (1999).

Friedman, Milton (1966), Capitalismo y libertad, Rialp, Madrid (1962).

Friedman, Milton (1970), «The Social Responsibility of Business Is to Increase Its Profits», New York Times Magazine, September 13, pp. 32-34.

Friedmann, Georges (1977), La crisis del progreso, Laia, Barcelona (1936).

Galbraith, John Kenneth (1967), The New Industrial State, Princenton University Press, Princenton.

Gaulejac, Vincent de (2005), La société malade de la gestion. Idéologie gestionnaire, pouvoir managérial et harcèlement social, Seuil, París.

Genoe McLaren, Patricia (2011), «James Burnham, The Managerial Revolution, and the development of management theory in post-war America », en Management E Organizational History, vol. 6, n. ${ }^{\circ} 4$, pp. 411-423.

Gramsci, Antonio (2000), «Americanismo y Fordismo», en Cuadernos de la cárcel, Tomo VI, Cuaderno 22, Era, México, pp. 59-95 (1975).

HAYEk, Friederich A. (2007), Camino de servidumbre, Alianza Editorial, Madrid (1944).

Inglehart, R. y Carballo, M. (2008), «¿Existe Latinoamérica? Un análisis global de diferencias transculturales», en Perfiles Latinoamericanos, n* ${ }^{\circ} 31$, pp. 13-38.

Jennings, Mariane M. (1999), «What's Happening in Business Schools», en Public Interest, n+ ${ }^{\circ} 137$, pp. 25-32.

Kakabadse Nada, K.; Cécile Rozuel y Linda Lee-Davies (2005), «Corporate social responsibility and stakeholder approach: a conceptual review», en 
International Journal of Business Governance and Ethics, vol. 1, núm 4, pp. 277-302.

Khurana, Rakesh (2007), From Higher Aims to Hired Hands. The Social Transformation of American Business Schools and the Unfulfilled Promise of Management as Profession, Princenton University Press, Princenton.

Knights, David y Glenn Morgan (1991), «Corporate Strategy, Organizations, and Subjectivityः A Critique», en Organization Studies, vol. 12, n+* 2, pp. 251273.

La Porta, Rafael; Florencio López-de-Silanes; Andrei Shleifer, y Robert Vishny (1999), «Corporate ownership around the world», en Journal of Finance, vol. 54, n. ${ }^{\circ}$, pp. 471-520.

Licha, Isabel (comp.) (2012), Enseñanza de la responsabilidad social empresarial. Retos de las universidades en Iberoamérica, Sudamericana, Buenos Aires.

Lourau, René (1975), El análisis institucional, Amorrortu, Buenos Aires (1970).

Martínez Rodríguez, Edim y Luis Ladislao García Martínez (2012), «Panorama de la enseñanza de responsabilidad social empresarial en las instituciones de educación superior en México e Iberoamérica», en Gestión y Estrategia, Universidad Autónoma Metropolitana-Azcapotzalco, n* 41, pp. 55-69.

Marris, Robin (1964), The Economic Theory of «Managerial» Capitalism, Free Press of Glencoe, Nueva York.

Mayo, Elton (1972), Problemas bumanos de una civilización industrial, Nueva Visión, Buenos Aires (1933).

McCraw, Harrison; Kathy S. Moffeit y John R. O’Malley Jr. (2009), «An Analysis of the Ethical Codes of Corporations and Business Schools», en Journal of Business Ethics, vol. 87, n+ ${ }^{\circ}$ 1, pp. 1-13.

Mintzberg, Henry (2004), Managers not MBA's. A hard look of the soft practice of managing and management development, Berrett-Koheler Publishers, San Francisco.

Mizruchi, Mark S. (2004), «Berle and Means Revisited: The Governance and Power of Large U.S. Corporations», en Theory and Society, vol. 33, núm 5, pp. 579-617.

Montaño Hirose, Luis (2000), «La transferencia de modelos organizacionales. Una propuesta analítica. El ejemplo de la Administración de la Calidad», en Administración y organizaciones, n. ${ }^{\circ}$, pp. 9-23.

- (2004), «Modernidad y cultura en los estudios organizacionales. Tres modelos analíticos», en Iztapalapa, pp. 15-33. 
- (2011), «El sentido de existencia social frente a los embates de la eficiencia», en Estudios Interdisciplinarios de la Organización, vol. 1, n. ${ }^{\circ}$ 1, pp. 35-49.

- (2012a), «Exploring the complex interaction between institutions and organizations», ponencia presentada en el 4th Latin American and European Meeting on Organization Studies, Ajijic, México.

- (2012b), «Organización, discurso y compromiso. Reflexiones acerca de los programas de responsabilidad social», en Esther Morales Franco, Gloria Nerty Navarro Castro y Martha Beatriz Santa Ana Escobar (cords.), Sustentabilidad, responsabilidad social y organizaciones. Dimensiones, retos y discursos, Remineo y Universidad Autónoma de Sinaloa, México, pp. 19-33.

Mullerat, Ramón (2007), En buena compañia. La responsabilidad social de las empresas, Debate, Barcelona.

Naciones Unidas (2010), Corporate Social Responsibility in Latin America, Nueva York y Ginebra, 144 pp.

North, Douglas C. (1993), Instituciones, cambio institucional y desempeño económico, en Fondo de Cultura Económica, México (1990).

Parsons, Talcott (1956), «Suggestions for a Sociological Approach to the Theory of Organizations I», en Administrative Science Quarterly, vol. 1, n. ${ }^{\circ} 1$, pp. 63-85.

PAsquero, Jean (2005), «La responsabilité sociale de l'entreprise comme objet des sciences de gestion: Un regard historique», en Marie-France B. Turcotте y Anne Salmon A. (eds.), Responsabilité sociale et environnementale de l'entreprise, Presses de l'Université du Québec, Quebec, pp. 80-111.

Polanyi, Karl (2009), El sustento del hombre, Capitán Swing, Madrid (1977).

Quairel-Lanoizelée, Françoise (2011), «ISO 26000, ou la “normalisation” de la responsabilité sociétale des organisations», en Gwenaëlle Nogatchewsky y Anne Pezet (coords.), Létat des enterprises 2012, Dauphine Recherches en Management, La Découverte, Paris, pp. 61-78.

Rendón Cobián, Marcela (2007), Dinámica organizacional y referentes institucionales. El caso de un pequeño hospital de propiedad familiar, Universidad Autónoma Metropolitana-Iztapalapa, México.

- (2012), «El papel de la estandarización en la responsabilidad social de las organizaciones. Del Fordismo a la norma ISO 26000», en Esther Morales Franco, Gloria Nerty Navarro Castro y Martha Beatriz Santa Ana Escobar (cords.), Sustentabilidad, responsabilidad social y organizaciones. Dimensiones, retos y discursos, Remineo y Universidad Autónoma de Sinaloa, México, pp. 61-83. 
Roe, Mark J. (1994), Strong Managers, Weak Owners, Princeton University Press, Princeton.

Simon, Herbert (1982), El comportamiento administrativo. Estudio de los procesos decisorios en la organización administrativa, Aguilar, Buenos Aires (1946).

Smith, Adam (1776), An Inquiry into the Nature and Causes of the Wealth of Nations, The Pennsylvania State University, 2005, Sitio Web: <http://www2. hn.psu.edu/faculty/jmanis/adam-smith/Wealth-Nations.pdf $>$.

TAYlor, Frederick Winslow (1961), Principios de la Administración Cientifica, Herrero Hermanos, México (1911).

Tylor, Edward Burnett (2009), Anábuac o México y los mexicanos antiguos y modernos, Universidad Autónoma Metropolitana-Iztapalapa y Juan Pablos, México (1861).

Weber, Max (1991), La ética protestante y el espiritu del capitalismo, Premia Editora, México (1905).

- (1970), Economía y Sociedad, Fondo de Cultura Económica, México (1922).

Williamson, Oliver (1989), Las instituciones económicas del capitalismo, Fondo de Cultura Económica, Mexico (1985).

Wooster, Harvey Alden (1919), «University Schools of Business and a New Business Ethics», en Journal of Political Economy, vol. 27, n. ${ }^{\circ}$ 1, pp. 47-63.

Young, A. P. (1939), «Social Responsibilities of Business: A Manager's View», en The Annals of the American Academy of Political and Social Science, $n .{ }^{\circ} 204$, pp. 86-92.

Zeitlin, Maurice (1974), «Corporate Ownership and Control: The Large Corporation and the Capitalist Class», en American Journal of Sociology, vol. 79, n. ${ }^{\circ}$ 5, pp. $1073-1119$. 